\title{
DESMITIFICANDO EL VALOR DE LAS IMÁGENES DE CELEBRIDADES EN SITIOS WEB DE ESTABLECIMIENTOS DE ALOJAMIENTO TURÍSTICO
}

\author{
Alejandro Alvarado Herrera \\ María Guadalupe Martínez Perezchica \\ Anastacio Gustavo Fernández Rodríguez \\ Universidad de Quintana Roo. México
}

\section{RESUMEN}

La presencia de celebridades en el diseño Web se considera valiosa por sus influencias en el atractivo visual y la credibilidad percibidos, así como en el tiempo dedicado por los usuarios de la Generación Y a ver los sitios. La investigación y las evidencias empíricas al respecto son escasas, lo que compromete la efectividad de su uso en páginas Web. Se hizo una investigación causal con un experimento verdadero y rastreo ocular en el que participaron 126 sujetos. Los hallazgos refutan esas ideas y revelan que la presencia de celebridades en esos contenidos puede ser contraproducente para las empresas.

Palabras clave: Rastreo ocular; atractivo visual; credibilidad; diseño Web; celebridades; marketing turístico.

\section{Demystifying the value of celebrities' images as source of value in accommodation promotion Websites}

\section{ABSTRACT}

The presence of celebrities' images on Websites is considered valuable since it is seen as a determinant factor of visual attractiveness, credibility and fixation time that Generation Y users assign to Websites. However, scientific research on this has been limited and empirical

Recibido: 11 de marzo de 2015

Devuelto para su revisión: 10 de junio de 2015

Aceptado: 30 de octubre de 2015

Departamento de Estudios Sociales y Empresariales. Universidad de Quintana Roo. Campus Cozumel. Avenida Andrés Quintana Roo s/n Esq. 110 Sur frente a la Colonia San Gervasio. C.P. 77600. Cozumel, QUINTANA ROO (México). E-mail: aah@uqroo.edu.mx, lupita.mtz.perezchica@gmail.com, werever2487@gmail.com 
evidence is scarce, which compromises the effectiveness of its use on Websites. Hence, a causal research, based on a true experiment and eye-tracking, was done. Findings refute those ideas and reveal that, surprisingly, the presence of celebrities in the content of Websites can be counterproductive for entrepreneurs.

Keywords: Eye-tracking; visual attractiveness; credibility; Website design; celebrities; tourism marketing.

\section{INTRODUCCIÓN}

Las tecnologías de la información y comunicación (TIC) han sido utilizadas por productores, inversionistas y consumidores para tomar decisiones. De acuerdo con el Foro Económico Mundial (2014), hay tres aspectos que explican la acelerada expansión en su uso: costos cada vez más accesibles; apertura de las redes de acceso y mayor interconexión humano-computadora. En este contexto, la red World Wide Web cobró una relevancia significativa para las compañías en las últimas décadas como herramienta para la difusión de productos y servicios y las páginas Web constituyen, frecuentemente, la primera impresión proyectada por una organización al mercado (Robins y Holmes, 2008). Luego, resulta fundamental para empresas y organizaciones contar con sitios Web con diseños efectivos, atractivos y verosímiles capaces de captar la atención, el interés y la confianza de la audiencia a la que están dirigidos dado al incremento de compradores y consumidores virtuales (Cyr et al., 2009; Lavie y Tractinsky, 2004).

Por otro lado, la demanda de los viajeros que se desplazan de región en región por diversos motivos ha ido evolucionando a tal punto que el turismo representa la segunda actividad económica mundial (Ritchie y Crouch, 2000; Sancho, 2006). Ante esta situación y considerando que la competencia que enfrentan las organizaciones de ese sector es cada vez mayor, es «destacable la aplicación intensiva de las nuevas tecnologías [...] [por lo que] las empresas turísticas cada día más se preocuparán por la satisfacción del usuario, que les exige una gestión de las posibilidades existentes para conocer sus preferencias» (Rubio, 2003:5). No sorprende entonces que el marketing sea una disciplina sobresaliente entre las empleadas para estudiar el turismo (Echtner y Jamal, 1997), ni tampoco que el diseño y difusión de páginas Web constituyan una de las principales herramientas para acceder a los consumidores potenciales, además de fungir como medio de gestión para las empresas turísticas, especialmente las de pernocta (Guttentag, 2009).

Un segmento de mercado de especial interés es el llamado Generación Y, el cual está conformado por individuos nacidos entre 1977-1994 (Sheahan, 2005). La importancia de este segmento de consumidores radica en que poseen particularidades relativas: a sus comportamientos, actitudes y habilidades en el uso del Internet y de los medios masivos de comunicación (Bakewell y Mitchell, 2003; Leask et al., 2014; Martínez, 2013; Roy et al., 2015), mayores ingresos, consumo y tiempo dedicado en navegar por la red (Waters, 2006), que han crecido de la mano con la innovación tecnológica (Djamasbi et al., 2007, 2008). Estas características se traducen en también mayores niveles de exigencia sobre el atractivo visual y la credibilidad de las páginas Web (Tractinsky, 2004; Williams y Page, 2011). 
A partir de los resultados de una investigación exploratoria, diseñada y realizada para determinar las características específicas que debe contener una página Web para ser considerada visualmente atractiva por usuarios de la Generación Y, Djamasbi, Siegel y Tullis (2010) postularon que son: i) una imagen principal grande; ii) poco texto; iii) una herramienta de búsqueda; y iv) presencia de celebridades. Si bien valiosos, esos planteamientos demandan mayor atención investigadora ya que, tal como señalan los propios Djamasbi et al. (2010), la evidencia empírica que los soporta es exigua y es que, en efecto, los estudios realizados en la investigación referida se basaron en muestras no probabilísticas y en un diseño pre-experimental apoyado con rastreo ocular sin grupo de control.

Luego, con el objetivo de someter a falsación dichos postulados y atendiendo a un contexto cultural distinto, se realizó un experimento verdadero apoyado también en el uso de dispositivos de rastreo ocular, empleando tres versiones diferentes de dos páginas Web de establecimientos de alojamiento turístico previamente seleccionadas y manipulando la presencia/ausencia de celebridades y de personas comunes en la pantalla para determinar las posibles diferencias en la influencia ejercida por cada una en el atractivo y credibilidad percibidos por los usuarios de páginas Web, así como el tiempo dedicado a observar (tiempo de fijación de la mirada) el área que contenía cada estímulo en específico.

La relevancia y pertinencia de este trabajo radica en varios aspectos. Por un lado, hasta donde sabemos, es la primera ocasión en que se realiza una investigación causal sobre el tema específico a través de un diseño experimental apoyado en rastreo ocular en el ámbito del marketing en general y en el del marketing turístico en particular, lo que constituiría, en consecuencia, una de sus contribuciones académicas principales. Por otro lado, se pretende aportar bases teóricas y empíricas que permitan determinar si realmente las celebridades constituyen una fuente de atractivo visual de páginas Web para el segmento específico; confirmar o refutar si sus percepciones acerca de la credibilidad de sitios Web están basadas en el diseño más que en el contenido y si se ven influenciadas por la presencia de personas, célebres o no. Finalmente, confirmar o refutar que el tiempo y atención que los usuarios dedican a observar las imágenes de celebridades o de personas no célebres incluidas en el diseño de las páginas Web de establecimientos de alojamiento turístico, constituye una fuente de ventaja competitiva para los anunciantes, o si, por el contrario, demerita la propia información que éstos desean proyectar al mercado. De esta forma, las contribuciones esperadas alcanzarían también al ámbito profesional, ya que los conocimientos generados ayudarían a los tomadores de decisiones a optimizar tanto el diseño de sus sitios Web, en términos funcionales y estéticos, como a evitar incurrir en gastos innecesarios, si fuera el caso.

En los apartados siguientes se detallan la revisión de la literatura y el desarrollo de las hipótesis correspondientes, el diseño metodológico y su implementación, el análisis y la discusión de los resultados y, finalmente, las implicaciones, limitaciones y líneas de investigación futuras identificadas.

\section{REVISIÓN DE LA LITERATURA}

La industria turística ha tenido un crecimiento notable en las últimas décadas (Ritchie y Crouch, 2000; Deng et al, 2002; Sancho, 2006) y no es ajena a los procesos propios de la globalización y del desarrollo de las TIC. Luego, resulta comprensible el auge del 
estudio del marketing turístico como un elemento de gestión y adaptabilidad a un mercado totalmente competitivo (Bigné, 1996). Ante esa dinámica social y de cambio, los directores de marketing se enfrentan a decisiones cada vez más complejas, tanto en lo referente al diseño de productos y servicios (Kotler, 2011) como en lo tocante a sus comunicaciones integradas de marketing, y es que por un lado, los medios de comunicación son cada vez más variados, complejos, fragmentados y saturados (Martí et al., 2010) y por otro, los consumidores han incrementado su selectividad, exigencia, sofisticación y renuencia a la publicidad (Torrado y Flores, 2007).

La atención a esta problemática supone un reto mayor para los responsables de las estrategias y campañas de comunicación comercial, pues la efectividad publicitaria y la satisfacción de las necesidades y expectativas de los anunciantes se ven comprometidas (Del Pino y Olivares, 2007). No obstante, el aprovechamiento adecuado de las TIC constituye un elemento útil y una oportunidad para afrontar el reto que supone (Buhalis y Law, 2008; Tang et al., 2011). En este sentido, los sitios Web han sido usados como uno de los principales canales de contacto, distribución de información y comunicación entre las empresas, sus consumidores y demás públicos, particularmente con los miembros de la Generación Y (Bakewell y Mitchell, 2003, Cui et al., 2003; Djamasbi et al., 2007, 2008, 2010; Leask et al., 2014; Martinez, 2013; Roy et al., 2015; Sheahan, 2005; Tractinsky, 2004 y Waters, 2006), quienes son altamente influenciados por el uso de imágenes y valoran la verdad, la integridad y la honestidad (Williams y Page, 2011) y constituyen los sujetos de estudio en esta investigación.

Así, la credibilidad de la información proyectada a través de las páginas de Internet es determinante para la efectividad del mensaje y para la evaluación del atractivo percibido por los usuarios, dado que la primera impresión de una página Web está basada en los elementos visuales y responde a juicios preconcebidos que tienen lugar incluso antes de que otros procesos cognitivos ocurran en la mente del consumidor (Robins y Holmes, 2008). En esta misma línea de pensamiento, otras investigaciones destacan que el uso de imágenes, sobre todo de personas y texto en un sitio Web, son capaces de provocar un clima más personalizado al generarse una respuesta emocional, e influir positivamente en la confianza y disfrute de los usuarios al navegar por el sitio de Internet (Cyr et al., 2009; Herrero y San Martín, 2012).

\subsection{Atractivo visual de páginas Web}

El término 'atractivo' ha sido abordado desde distintas perspectivas a lo largo de la historia, desde los filósofos de la Grecia Antigua hasta su uso moderno como instrumento de marketing (Lavie y Tractinsky, 2004) y está estrechamente relacionado con el placer, la belleza y la armonía que los seres humanos son capaces de experimentar (Lindgaard, 2007). En el contexto de esta investigación, el atractivo visual es concebido y usado para denotar todo aquello «que puede ser llamado 'estético'» (Lindgaard et al., 2006:117). En otras palabras, el atractivo visual es una respuesta estética de los sujetos ante un objeto dado, y es posible identificar dos enfoques principales en la literatura para investigarlo.

El primero de esos enfoques, basado en la teoría Gestalt (Arnheim, 1988, 1992), postula que las percepciones de los individuos acerca de un objeto determinado son glo- 
bales y que deben estudiarse a partir de estímulos completos y naturales, mientras que el segundo se basa en la estética experimental y propugna que es posible descubrir de una mejor manera las percepciones de los sujetos a través de los efectos producidos por partes aisladas de un objeto, mediante la experimentación (Martindale et al., 1990; Swede, 1994). En este trabajo, de forma análoga a lo establecido por Djamasbi et al. (2010), se optó por aprovechar ambos enfoques a través de un cuestionario tradicional de encuesta y un experimento apoyado con tecnología de rastreo ocular, respectivamente.

Tal como se señaló, una de las cuatro características identificadas como determinantes del atractivo visual declarado por usuarios de páginas Web -pertenecientes a la Generación Y- es la presencia de celebridades en ellas (Djamasbi et al., 2010). Si bien esto podría sugerir la necesidad de plantear una hipótesis que estableciera una relación teórica de dependencia entre la presencia de celebridades en el estímulo y el atractivo visual del mismo, dadas: i) la naturaleza exploratoria del trabajo previo, ii) la escasa evidencia empírica de soporte y iii) la necesidad de evitar cometer un error tipo II, se plantea que, por el contrario:

$\mathrm{H}_{1}$ : El atractivo visual declarado por usuarios de la Generación Y de una página Web de promoción de alojamiento turístico no difiere significativamente por la presencia de celebridades o de personas no célebres en su diseño.

\subsection{Credibilidad en entornos virtuales}

La vasta cantidad y la facilidad de acceso a la información de uso público vía Internet, aunadas a la libertad creativa sobre la forma y los contenidos de los mensajes comerciales en los medios digitales, permiten el desarrollo de espacios únicos en la búsqueda de ventajas comparativa y competitiva que los profesionales del marketing requieren para la promoción de los servicios y productos de sus firmas, dada su creciente importancia en el proceso de toma de decisiones de los consumidores (Pitta, 2011). Sin embargo, también se ha facilitado la posibilidad de producir y difundir versiones con alteraciones, malas representaciones e incluso falsificaciones de dichos mensajes, aumentando así la preocupación y la necesidad de asegurar la credibilidad, ya que el origen, la calidad y la veracidad de la información, se han vuelto más difusos de lo que nunca antes fueron (Metzger y Flanagin, 2013; Sundar, 2008).

El término 'credibilidad' puede ser concebido desde distintos enfoques, no obstante, sólo dos de ellos han sido considerados de especial interés en el marco de esta investigación. El primero, surge de las áreas de la psicología y la comunicación, y su énfasis es en la fuente, en virtud de la importancia que la integridad del emisor tiene en la conformación de la credibilidad, y está, en consecuencia, estrechamente relacionado con la noción aristotélica del ethos, es decir, se basa en el carácter del emisor. Por otro lado, desde el ámbito de las ciencias informáticas, el estudio de la credibilidad ha tomado como objeto de atención el contenido del mensaje más que la fuente emisora del mismo, obedeciendo a la noción filosófica del logos, es decir, la que se basa en la lógica y la razón (Metzger y Flanagin, 2013). 
En este sentido, la credibilidad en entornos virtuales se refiere al grado en que los usuarios confían en la información contenida en un sitio Web (Robins y Holmes, 2008), y se sustenta en los juicios que hacen en relación con la fuente de la información y con el mensaje en sí mismo (Sundar, 2008). Adicionalmente, algunas investigaciones han encontrado que los usuarios suelen evaluar la credibilidad de las páginas Web basándose en los elementos visuales contenidos en su diseño más que en sus contenidos o fuente de la información (Fogg et al., 2003), por lo que recomiendan a los anunciantes asegurarse de invertir en un diseño que resulte altamente atractivo para el usuario en aras de lograr que un sitio Web transmita confianza amplia.

En apego a lo anterior, Cyr y colaboradores (2009), argumentan que la presencia de imágenes y de texto en una página Web, pueden impactar positivamente en la credibilidad percibida por los usuarios, específicamente y en mayor medida, las imágenes de personas, por lo cual éstas pueden ser usadas para obtener respuestas emocionales de calidez y confiabilidad en entornos virtuales, procurando una actitud más favorable de los consumidores hacia el sitio Web. Cabe aclarar que dada su relevancia en los entornos virtuales, se decidió incluir esta variable adicional en la investigación, lo cual constituye una aportación en sí misma toda vez que no fue considerada en el documento base sometido a falsación (Djamasbi et al., 2010). Según estos postulados la credibilidad percibida por los usuarios es influida directa y significativamente por el uso de imágenes de personas en las páginas Web. No obstante, de forma análoga a lo establecido en torno al atractivo visual, dada la escasa evidencia empírica al respecto y procurando evitar la comisión de un error tipo II, se plantea que:

$\mathrm{H}_{2}$ : La credibilidad declarada por usuarios de la Generación Y de una página Web de promoción de alojamiento turístico no difiere significativamente por la presencia de celebridades o de personas no célebres en su diseño.

\subsection{Tiempo de fijación de la mirada}

La investigación del movimiento ocular comprende, de acuerdo con Rayner (1998), tres etapas: la inicial (1879-1920) referente a las primeras observaciones acerca de la rapidez con que se mueve el ojo o sobre los movimientos sacádicos al leer textos; la segunda (19201970) enfocada en la investigación del proceso cognitivo para entender el comportamiento del individuo; y la última (1970-2014), derivada de los avances tecnológicos en la materia y la creación sistemas de rastreo ocular más poderosos, sofisticados y económicos, centrada en el procesamiento y el análisis de la información recabada (Jacob y Karn, 2003) para su uso en diversas áreas del conocimiento, principalmente en los estudios psicológicos. Una revisión de 21 investigaciones asistidas con métodos de rastreo ocular pertenecientes a esta tercera etapa señala que las variables empleadas de forma más recurrente son:

Número de fijaciones, duración promedio de la fijación, número de fijaciones en cada área de interés, proporción de tiempo empleado en cada área de interés, duración promedio de la mirada en cada área de interés y el índice general de fijación (Jacob y Karn, 2003:584). 
El tiempo de fijación de la mirada se refiere al espacio temporal que destina un individuo a observar un objeto determinado o, como en este caso, a un área de interés dada. La información arrojada por un sistema de rastreo ocular incluye el registro de los movimientos sacádicos y el tiempo de fijación de ambos ojos (Lai et al., 2013). Los datos registrados del tiempo de fijación suelen representarse a través de mapas de calor y mapas de fijación, los cuales permiten identificar periodos de fijación, altos o bajos, que destina un sujeto a un objeto en específico (Djamasbi et al., 2008).

Es oportuno señalar que existen diversas opiniones respecto al tiempo de fijación mínimo requerido para que pueda considerarse apreciable. Al respecto, Cyr y Head (2013) establecen que ese tiempo debe ser mayor que $500 \mathrm{~ms}$ para poder evaluar de forma significativa un contenido del sitio Web; Rayner señala que «nuestros ojos se mantienen relativamente quietos durante fijaciones de entre $200 \mathrm{~ms}$ y $300 \mathrm{~ms}$ (1998:373); y finalmente, Djamasbi et al. (2007) sostienen que para considerarse fijación debe exceder los $300 \mathrm{ms,}$ siendo éste el tiempo mínimo considerado como una fijación en el presente trabajo con el objeto de procurar la comparabilidad de resultados.

Las tecnologías de rastreo ocular están siendo ampliamente utilizadas para estudiar y entender aquellas áreas de páginas Web (Buscher et al., 2009; Roth et al., 2013), tanto de búsqueda como de promoción, en las que los usuarios invierten más atención y el orden en que lo hacen (Guan y Cutrell, 2007). De manera particular, en lo correspondiente al uso de motores de búsqueda de páginas Web, Buscher et al. (2010) demostraron que la atención de los usuarios depende de la calidad y el contenido de otros elementos en las páginas Web, tales como los anuncios. Por su parte, Dumais et al. (2010), determinaron que los usuarios de páginas Web de búsqueda dedican mayor atención visual a los tres primeros resultados y a los anuncios principales arrojados por el buscador, brindando menor atención a otras áreas de la página, clasificando a los usuarios en tres tipos de acuerdo al tiempo de fijación dedicado a cada área de interés: exhaustivo, económico enfocado en los resultados, y económico con atención prioritaria en los anuncios.

Los resultados del rastreo ocular en el experimento realizado por Cutrell y Guan (2007), sugieren que la diferencia en la ejecución de los buscadores Web se debe a que, a medida que aumenta la longitud de los fragmentos descriptivos de las páginas Web, los usuarios prestan más atención a esa descripción en detrimento de la dirección URL localizada en la parte inferior del resultado de cada búsqueda. De igual manera, determinaron que los usuarios observan los resultados de alguna búsqueda siguiendo un orden más o menos lineal, donde la mayor actividad de la mirada se dirigió a los primeros objetos visualizados en la pantalla, en tanto aquellos localizados más abajo, reciben cada vez menor atención.

El uso de sistemas de rastreo ocular como herramienta de apoyo en el diseño de mensajes comerciales en general, y para el desarrollo del contenido de páginas Web en particular, permite crear ciertas ventajas competitivas e incrementar el valor de las organizaciones, dado que propicia un aprovechamiento máximo de los elementos funcionales -entendidos como los relativos a la utilidad percibida del producto o servicio ofertado-, y hedónicos -aquellos de naturaleza estética y enfocados en aspectos afectivos o emocionales- que lo componen, optimizando así el diseño de sus sitios Web (Djamasbi et al., 2007) y generando confianza en el consumidor (Cyr y Head, 2013). 
De acuerdo al paradigma Estimulo-Organismo-Reacción (Jacoby, 2002), las reacciones del consumidor ante el estímulo presentado se basan más en su situación afectiva y emocional, lo que puede suponer que los usuarios podrían dedicar más atención a las zonas hedónicas que a las funcionales de la página Web con consecuencias indeseables para los anunciantes, pues se conoce que el uso excesivo de estímulos en el diseño "podría ejercer una influencia negativa sobre el tiempo y dinero que gasta el consumidor [...] afectando la efectividad de la publicidad del sitio Web» (Wang et al., 2007:383). Otras investigaciones señalan que tanto la complejidad como el exceso de información de los sitios Web tales como la utilización de colores demasiado brillantes y gráficos animados pueden constituir elementos distractores capaces de provocar dificultad para el usuario al navegarlos (Huang, 2003) y producir un efecto negativo sobre la atención del consumidor (Zhang, 2000). En este contexto se plantea que:

$\mathrm{H}_{3}$ : La incorporación de elementos gráficos no relacionados con la empresa en sí misma incrementa el tiempo de fijación de la mirada sobre los mismos y distrae la atención del consumidor.

\section{METODOLOGÍA}

Con el objetivo de testear hallazgos de investigaciones previas en torno a las características determinantes del atractivo visual y la credibilidad declarados por usuarios de la Generación Y de páginas Web de promoción de establecimientos de alojamiento turístico, se diseñó y llevó a cabo una investigación causal basada en un experimento verdadero apoyado en un cuestionario de encuesta y tecnología de rastreo ocular en un contexto cultural distinto a aquel en que fueron desarrolladas, como se describe a continuación.

Para realizar el diseño experimental para un factor 3 x 2 sólo post-test mostrado en la Tabla 1, se usaron tres versiones diferentes de dos páginas Web de alojamientos turísticos exóticos previamente seleccionadas, en las cuales se manipuló el factor presencia/ausencia de celebridades y de personas no célebres en la pantalla para los grupos sujetos a los tratamientos y no así para los del grupo de control, para determinar las posibles diferencias en la influencia ejercida por cada una en las variables dependientes atractivo visual y credibilidad percibidos de las páginas Web, así como en el tiempo de fijación de la mirada en el área que

Tabla 1

ESTRUCTURA DE DISEÑO EXPERIMENTAL

\begin{tabular}{lccc}
\hline Grupo & Selección & Tratamiento & $\begin{array}{c}\text { Observación } \\
\text { Post-test }\end{array}$ \\
\hline $\mathrm{GE}_{1}$ & $(\mathrm{R})$ & $\mathrm{X} 1$ & 01 \\
$\mathrm{GE}_{2}$ & $(\mathrm{R})$ & $\mathrm{X} 2$ & 02 \\
$\mathrm{GC}$ & $(\mathrm{R})$ & & 03 \\
\hline$(\mathrm{R})=$ Aleatoria & & &
\end{tabular}


contenía cada estímulo. Dicho diseño fue concebido y aplicado atendiendo tanto a la naturaleza de la investigación como a la necesidad de procurar la comparabilidad de sus resultados.

El trabajo comenzó con la selección de dos páginas Web de promoción de establecimientos de alojamiento turístico exóticos rurales a través del portal www.booking.com, el sitio de búsqueda de servicios relacionados al turismo más utilizado a nivel global según el Reporte Alexa (2013). Al incluir como criterio de búsqueda la palabra 'rural', el principal destino desplegado por dicha fuente fue 'Ubud', localizado en Bali, Indonesia, arrojando un total de 80 hoteles, de los cuales se eligieron sólo las dos páginas Web mejor y peor posicionadas según las puntuaciones otorgadas por los usuarios, que cumplieran además, con el criterio de tener tres de las cuatro características visuales consideradas determinantes del atractivo visual para la Generación Y: imagen principal grande, poco texto y una herramienta de búsqueda (Djamasbi et al., 2010); correspondiendo a las páginas Web que a partir de este punto se designarán como 'pw1' y 'pw2', respectivamente.

El hecho de que no tuvieran presencia de celebridades o de personas no célebres fue un criterio esencial para poder realizar la manipulación del factor de forma precisa y poder contar con un grupo de control sujeto a los estímulos originales sin modificaciones. El diseño de los estímulos se realizó como sigue: el correspondiente al grupo de control (GC) contuvo las dos páginas Web tal y como se encontraron publicadas; posteriormente se añadió, a cada página, una imagen de celebridades para el grupo experimental $1\left(\mathrm{GE}_{1}\right)$ y una imagen de personas no célebres con características físicas y actitudinales similares para el grupo experimental $2\left(\mathrm{GE}_{2}\right)$.

Se realizó una prueba piloto con 32 miembros de la Generación Y seleccionados aleatoriamente de la población universitaria, siendo ésta su única participación en el experimento, con el fin de verificar que la manipulación de los estímulos fuera adecuada en términos de calidad, pertinencia y reconocimiento de las celebridades incorporadas. Este último aspecto fue confirmado a través de la realización de dos preguntas abiertas diseñadas específicamente para tal fin, las cuales fueron incluidas en el cuestionario aplicado a los participantes de la prueba piloto. Los resultados obtenidos fueron adecuados toda vez que los sujetos expuestos a la imagen que incluyó a las celebridades las identificaron positivamente.

Posteriormente, se presentó una de las tres versiones de cada página Web a otros 94 estudiantes universitarios seleccionados aleatoriamente en Cozumel, Quintana Roo, México, cuyas edades estaban en el rango propuesto para la Generación Y, siendo distribuidos equitativamente, también de forma aleatoria, en cada uno de los tres grupos establecidos. Es importante destacar que los usuarios seleccionados participaron de forma voluntaria tras ser debidamente informados sobre el procedimiento experimental, así como el procesamiento anónimo y agrupado que tendrían los datos, y de esta manera, teniendo además perfecta claridad en sus dudas e inquietudes, firmaron una carta de consentimiento expreso.

El rastreo ocular y la captura de los datos se realizaron utilizando el dispositivo Gazepoint GP3 y su software Gazepoint Analysis (v.2.2.0). La decisión de usar dicho equipo obedeció a las razones siguientes: en primer lugar destaca que el ángulo visual del Gazepoint GP3 $\left(0.5^{\circ}\right.$ a $\left.1.0^{\circ}\right)$ permite determinar adecuadamente las fijaciones de la vista (Barber et al., 2014); en segundo lugar, que al tratarse de un sistema remoto (Fong et al., 2015) que se monta directamente en la pantalla en la que se proyectan los estímulos no invasivo para los participantes; en tercer lugar, que hay evidencia empírica que soporta 
que la precisión de dicho dispositivo es adecuada para el tipo de investigación en cuestión (Zugal y Pinggera, 2014); y, finalmente, que se trata de uno de los nuevos dispositivos que ofrecen soporte de rastreo ocular a un coste razonable comparado con otras tecnologías (Zugal y Pinggera, 2014).

El tiempo de proyección de cada página Web fue de 10 segundos por considerarse suficiente para generar una percepción clara (Djamasbi et al., 2010). Se utilizó además un fondo de pantalla negro antes y después de la proyección de cada página con la finalidad de no distraer la atención de los sujetos en ningún momento (Robins y Holmes, 2008), evitando así sesgar sus percepciones (Lindgaard, 2007). Los factores externos tales como los niveles de iluminación, temperatura y humedad ambientales y el confort y la postura de los participantes fueron controlados en todo momento para asegurar la validez del experimento.

En lo relativo al atractivo visual y la credibilidad percibidos por los sujetos acerca de las dos páginas Web a las que fueron expuestos, se diseñó un instrumento de medición a través de un cuestionario de encuesta autoadministrado, el cual se aplicó a los usuarios de forma individual al terminar la proyección de los estímulos que les correspondieron. La escalas de medición que sirvieron como base para la elaboración del cuestionario fueron: Actitud hacia el sitio Web (Atractivo visual), desarrollada por Mattwick, Malhotra, y Rigdon $(2001,2002)$, la cual pretende medir, a través de 3 ítems precedidos de la afirmación «En mi opinión sobre el atractivo visual considero que....» (... la forma en que la página Web muestra sus productos es atractiva / ... la página Web es estéticamente atractiva / ... me gusta la forma en que luce la página Web), qué tan atractivo visualmente cree una persona que resulta un sitio Web (Bruner II, 2009); y la escala de Credibilidad de Sinclair e Irani (2005), que intenta medir la credibilidad de una compañía o anunciante, a través de 4 ítems precedidos de la afirmación: «Con respecto a la credibilidad en mi opinión....» (... confío en la página Web / ... la página Web hace declaraciones verdaderas / ... la página Web es honesta / ... creo en lo que me dice la página Web).

Cabe mencionar, que se decidió no otorgar definición alguna de los constructos atractivo visual y credibilidad a los participantes para evitar introducir sesgos en sus respuestas. De hecho, ambas escalas fueron seleccionadas por haber demostrado poseer propiedades psicométricas apropiadas, provenir de revistas científicas de marketing del más alto nivel, tener una buena adecuación al objeto de estudio de este trabajo y ser parsimoniosas. En todos los casos se empleó un formato tipo Likert con 7 puntos de asignación de respuesta, anclados 1 Totalmente en desacuerdo y 7 Totalmente de acuerdo. Los datos obtenidos tanto a través del dispositivo de rastreo ocular como los de la encuesta fueron analizados estadísticamente mediante SPSS y sus resultados se presentan en la sección siguiente.

\section{ANÁLISIS Y DISCUSIÓN DE LOS RESULTADOS}

\subsection{Datos demográficos y fiabilidad de las escalas empleadas}

Los datos demográficos de los 94 voluntarios participantes en el experimento se detallan a continuación en la Tabla 2. Como puede observarse, $56.4 \%$ de los sujetos fueron hombres y $43.6 \%$ mujeres, todos pertenecientes a la Generación Y, correspondiendo $85.1 \%$ de ellos al rango de edades comprendidas entre 18 y 24 años y $14.9 \%$ entre 25 y 31 años. 


\section{Tabla 2 \\ DATOS DEMOGRÁFICOS}

\begin{tabular}{ll}
\hline $\mathrm{N}$ & 94 participantes \\
Sexo & \\
$\quad$ Mujeres & $43.6 \%$ \\
$\quad$ Hombres & $56.4 \%$ \\
Edad & \\
$\quad$ Entre 18 y 24 años & $85.1 \%$ \\
$\quad$ Entre 25 y 31 años & $14.9 \%$ \\
\hline
\end{tabular}

En cuanto a la fiabilidad de las escalas de medición de atractivo visual y credibilidad se refiere, ambas demostraron poseer un desempeño considerablemente superior al valor crítico comúnmente aceptado $(\alpha>0.7)$ cada una de las veces que fueron empleadas. Como se aprecia en la Tabla 3, la más baja fue la correspondiente a la medición del atractivo visual de la página Web $1\left(\alpha_{\mathrm{pw}}>0.871\right)$ y la más alta a la medición de la credibilidad de la página Web $2\left(\alpha_{\mathrm{pw} 2}>0.936\right)$.

\section{Tabla 3}

\section{FIABILIDAD SIMPLE DE LAS ESCALAS DE MEDIDA}

\begin{tabular}{lc}
\hline Variable/ Página Web & $\boldsymbol{\alpha}$ \\
\hline Atractivo visual /pw1 & .871 \\
Atractivo visual /pw2 & .886 \\
Credibilidad / pw1 & .909 \\
Credibilidad / pw2 & .936 \\
\hline
\end{tabular}

\subsection{Contrastación de hipótesis}

En la Tabla 4 se consignan los estadísticos descriptivos para las variables atractivo visual y credibilidad percibidos de cada una de las dos páginas Web proyectadas al grupo de sujetos.

Como se muestra, todos los valores medios del atractivo visual oscilan entre $\mu$ ATRACTIVO_VISUAL GCpw2 $_{2}=4.767$ y $\mu$ ATRACTIVO_VISUAL GElpw $_{2}=4.873$, valores mínimo y máximo correspondientes a las opiniones de los sujetos del GC y las del $\mathrm{GE}_{1}$ respectivamente, ambos sobre la página Web 2 . El hecho de que todas las medias tienden a 5 sugiere que los usuarios están de acuerdo en que ambas páginas Web les resultan atractivas. En cuanto a la credibilidad se refiere, los valores medios mínimo y máximo son

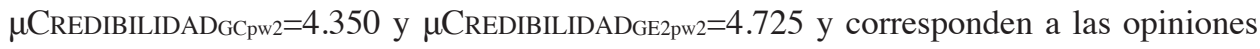
de los grupos $\mathrm{CG}$ y $\mathrm{GE}_{2}$ acerca de la misma página Web 2, predominando valores en torno a 4.5 y tendiendo también a estar de acuerdo en que la información contenida en las dos páginas es verosímil. 


\section{Tabla 4 \\ DESCRIPTIVOS DE ATRACTIVO VISUAL Y CREDIBILIDAD, POR PÁGINA WEB}

\begin{tabular}{|c|c|c|c|c|c|c|c|c|}
\hline \multirow{2}{*}{$\begin{array}{c}\text { Variable / } \\
\text { Página Web }\end{array}$} & \multirow{2}{*}{ Grupo } & \multirow{2}{*}{$\mathbf{N}$} & \multirow{2}{*}{ Media } & \multirow{2}{*}{$\begin{array}{l}\text { Error } \\
\text { estándar }\end{array}$} & \multicolumn{2}{|c|}{$\begin{array}{c}\text { Intervalo de } \\
\text { confianza para la } \\
\text { media al } 95 \%\end{array}$} & \multirow[t]{2}{*}{ Min } & \multirow[t]{2}{*}{ Max } \\
\hline & & & & & $\begin{array}{l}\text { Lim. } \\
\text { inf. }\end{array}$ & $\begin{array}{l}\text { Lim. } \\
\text { sup. }\end{array}$ & & \\
\hline \multirow{4}{*}{$\begin{array}{l}\text { Atractivo visual / } \\
\text { pw1 }\end{array}$} & $\mathrm{GE}_{1}$ & 34 & 4.784 & 0.199 & 4.378 & 5.190 & 1.33 & 6.67 \\
\hline & $\mathrm{GE}_{2}$ & 30 & 4.867 & 0.224 & 4.410 & 5.324 & 2.00 & 6.67 \\
\hline & GC & 30 & 4.856 & 0.189 & 4.469 & 5.242 & 1.00 & 6.67 \\
\hline & Total & 94 & 4.833 & 0.117 & 4.601 & 5.065 & 1.00 & 6.67 \\
\hline \multirow{4}{*}{$\begin{array}{l}\text { Atractivo visual / } \\
\text { pw2 }\end{array}$} & $\mathrm{GE}_{1}$ & 34 & 4.873 & 0.231 & 4.402 & 5.343 & 1.00 & 7.00 \\
\hline & $\mathrm{GE}_{2}$ & 30 & 4.800 & 0.221 & 4.371 & 5.229 & 2.33 & 6.67 \\
\hline & GC & 30 & 4.767 & 0.226 & 4.305 & 5.228 & 1.00 & 7.00 \\
\hline & Total & 94 & 4.816 & 0.128 & 4.562 & 5.069 & 1.00 & 7.00 \\
\hline \multirow{4}{*}{$\begin{array}{l}\text { Credibilidad / } \\
\text { pw1 }\end{array}$} & $\mathrm{GE}_{1}$ & 34 & 4.537 & 0.203 & 4.124 & 4.950 & 2.00 & 7.00 \\
\hline & $\mathrm{GE}_{2}$ & 30 & 4.550 & 0.187 & 4.168 & 4.932 & 3.00 & 6.75 \\
\hline & GC & 30 & 4.358 & 0.202 & 3.945 & 4.771 & 1.00 & 6.50 \\
\hline & Total & 94 & 4.484 & 0.114 & 4.258 & 4.710 & 1.00 & 7.00 \\
\hline \multirow{4}{*}{$\begin{array}{l}\text { Credibilidad / } \\
\text { pw2 }\end{array}$} & $\mathrm{GE}_{1}$ & 34 & 4.552 & 0.201 & 4.143 & 4.960 & 2.50 & 7.00 \\
\hline & $\mathrm{GE}_{2}$ & 30 & 4.725 & 0.191 & 4.333 & 5.115 & 3.25 & 7.00 \\
\hline & GC & 30 & 4.350 & 0.210 & 3.921 & 4.779 & 1.00 & 6.50 \\
\hline & Total & 94 & 4.543 & 0.116 & 4.313 & 4.772 & 1.00 & 7.00 \\
\hline
\end{tabular}

Ahora bien, como se muestra en la Tabla 5, los resultados de los ANOVA realizados para la contrastación de las primeras dos hipótesis -las cuales establecen $\mathrm{H}_{1}$ : el atractivo visual declarado por usuarios de la Generación Y de una página Web de promoción de alojamiento turístico no difiere significativamente por la presencia de celebridades o de personas no célebres en su diseño; y $\mathrm{H}_{2}$ la credibilidad declarada por usuarios de la Generación Y de una página Web de promoción de alojamiento turístico no difiere significativamente por la presencia de celebridades o de personas no célebres en su diseño respectivamente- las soportan con respecto a cada una de las páginas Web utilizadas en esta investigación.

En primer lugar, en lo relativo a $\mathrm{H}_{1}$, no existen diferencias significativas entre las opiniones medias de los sujetos de los tres grupos acerca del atractivo visual de la página Web 1 ( $\mu$ ATRACTIVO_VISUALGE1pw1 $=4.784$; $\mu$ ATRACTIVO_VISUALGE2pw1 $=4.867$ y $\mu$ ATRACTIVO_VISUALGCpw1 $=4.856)(p=0.952)$ ni tampoco sobre el atractivo visual de la página Web 2 ( $\mu$ ATRACTIVO_VISUALGE1pw2 $=4.873 ; \mu$ ATRACTIVO_VISUALGE1pw2 $=4.800 \mathrm{y}$ $\mu$ ATRACTIVO_VISUALGCpw2 $=4.767)(p=0.941)$, superando en ambos casos de forma clara el valor crítico comúnmente aceptado en la literatura $(p>0.05)$ por lo que la hipótesis 


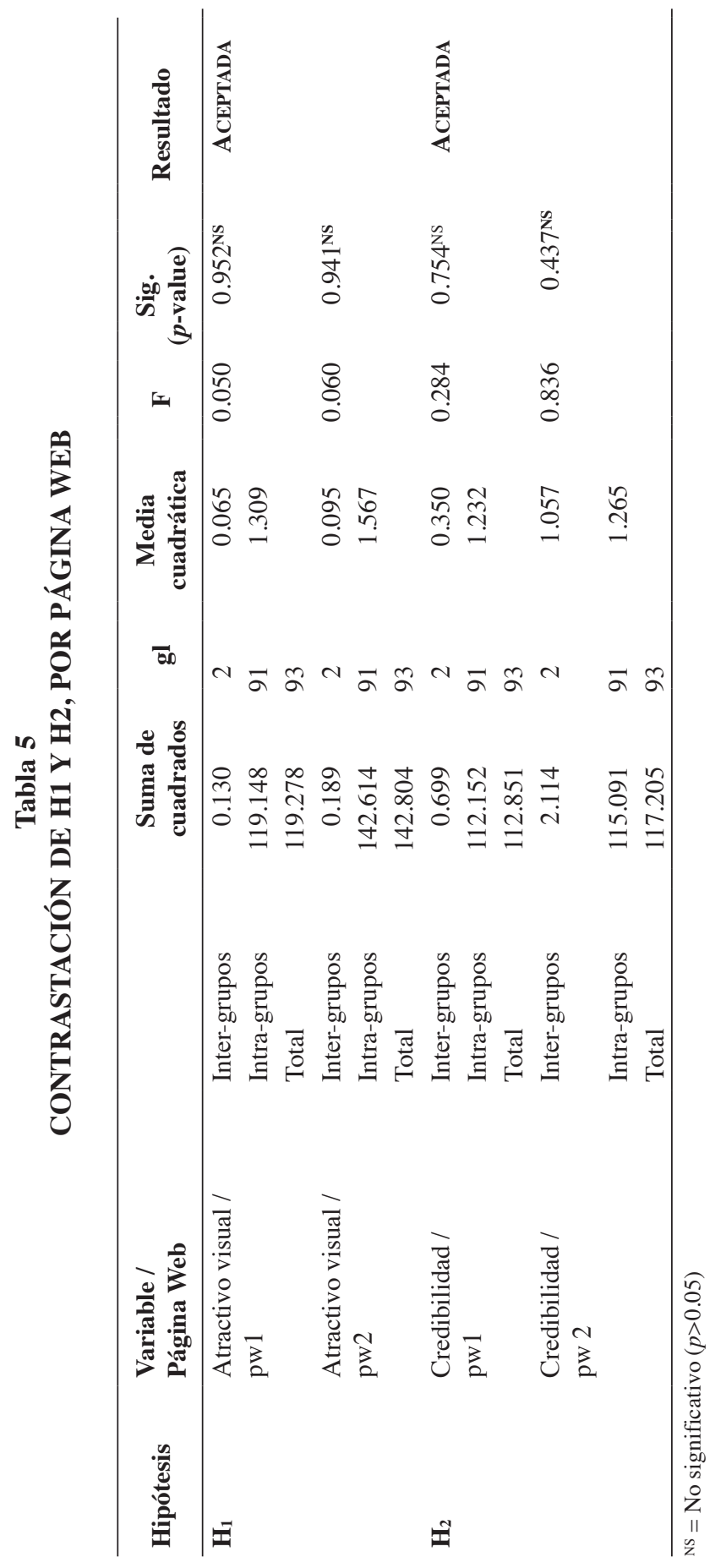


no puede ser rechazada y se confirma que: el atractivo visual declarado por usuarios de la Generación Y de una página Web de promoción de establecimientos de alojamiento turístico no difiere significativamente por la presencia de celebridades o de personas no célebres en su diseño.

En segundo lugar, en lo referente a $\mathrm{H}_{2}$, tampoco se hallaron diferencias estadísticamente significativas entre las opiniones medias de los sujetos de los tres grupos de estudio acerca de la credibilidad de la página Web $1\left(\mu\right.$ CREDIBILIDADGE $1 \mathrm{pw} 1_{1}=4.537$; $\mu$ RREDIBILIDAD $_{\mathrm{GE} 2 \mathrm{pw} 1}=4.550$ y $\mu$ RREDIBILIDADGCpwl $\left.=4.358\right)(p=0.754)$ ni tampoco

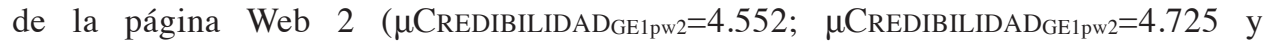
$\mu$ RREDIBILIDADGCpw2 $=4.350)(p=0.437)$, superandode igual forma el valor crítico establecido en la literatura $(p>0.05)$ y confirmando que: la credibilidad declarada por usuarios de la Generación Y de una página Web de promoción de establecimientos de alojamiento turístico no difiere significativamente por la presencia de celebridades o de personas no célebres en su diseño.

Por otro lado, con respecto al tiempo dedicado a observar el área que incluía cada uno de los estímulos utilizados en las páginas Web proyectadas, con la finalidad de contrastar la tercera hipótesis -la cual establece que $\mathrm{H}_{3}$ : la incorporación de elementos gráficos no relacionados con la empresa en sí misma incrementa el tiempo de fijación de la mirada sobre los mismos y distrae la atención del consumidor-, se obtuvieron los resultados que se puntualizan a continuación y que se muestran en la Tabla 6.

\section{Tabla 6}

DESCRIPTIVOS DEL TIEMPO DE FIJACIÓN EN EL ÁREA DE INTERÉS, POR PÁGINA WEB

\begin{tabular}{|c|c|c|c|c|c|c|c|c|}
\hline \multirow{2}{*}{$\begin{array}{l}\text { Variable / } \\
\text { Página Web }\end{array}$} & \multirow[t]{2}{*}{ Grupo } & \multirow[t]{2}{*}{$\mathbf{n}$} & \multirow[t]{2}{*}{ Media } & \multirow{2}{*}{$\begin{array}{c}\text { Error } \\
\text { estándar }\end{array}$} & \multicolumn{2}{|c|}{$\begin{array}{c}\text { Intervalo de confianza } \\
\text { para la media al } 95 \%\end{array}$} & \multirow[t]{2}{*}{ Min } & \multirow[t]{2}{*}{ Max } \\
\hline & & & & & Lim.inf. & Lim. sup. & & \\
\hline \multirow{4}{*}{$\begin{array}{l}\text { Tiempo de } \\
\text { fijación / } \\
\text { pw1 }\end{array}$} & $\mathrm{GE}_{1}$ & 34 & 1.067 & 0.126 & 0.812 & 1.325 & 0.06 & 3.55 \\
\hline & $\mathrm{GE}_{2}$ & 30 & 0.899 & 0.153 & 0.587 & 1.212 & 0.06 & 3.41 \\
\hline & GC & 30 & 0.221 & 0.071 & 0.077 & 0.367 & 0.00 & 1.33 \\
\hline & Total & 94 & 0.768 & 0.079 & 0.587 & 0.901 & 0.00 & 3.55 \\
\hline \multirow{4}{*}{$\begin{array}{l}\text { Tiempo de } \\
\text { fijación / } \\
\text { pw2 }\end{array}$} & $\mathrm{GE}_{1}$ & 34 & 1.001 & 0.102 & 0.793 & 1.209 & 0.19 & 2.83 \\
\hline & $\mathrm{GE}_{2}$ & 30 & 1.076 & 0.142 & 0.785 & 1.367 & 0.00 & 2.35 \\
\hline & GC & 30 & 0.340 & 0.062 & 0.052 & 0.305 & 0.00 & 1.17 \\
\hline & Total & 94 & 0.717 & 0.074 & 0.616 & 0.909 & 0.00 & 2.83 \\
\hline
\end{tabular}

Como puede observarse, el rango de valores adoptados por las medias de los tiempos de fijación de los tres grupos de estudio para ambas páginas Web es amplio, oscilando entre $\mu$ TIEMPO_DE_FIJACIÓNGCpwl=0.221 y $\mu$ TIEMPO_DE_FIJACIÓNGElpwl $=1.067$ segundos para la página Web mejor posicionada, es decir, la página Web 1 y entre $\mu$ TIEMPO_DE_FIJACIÓNGCpw2 $=0.340$ y $\mu$ TIEMPO_DE_FIJACIÓNGE1pw1 $=1.076$ segundos para la página Web 2 . 
Los resultados de los ANOVA correspondientes mostraron la existencia de diferencias estadísticamente significativas para los tiempos de fijación para $p<0.01$ por lo que, para completar la contrastación de $\mathrm{H}_{3}$, se llevaron a cabo los análisis de comparaciones múltiples necesarios, cuyos principales resultados se muestran en la Tabla 7.

Tabla 7

RESULTADOS DE LOS ANÁLISIS DE COMPARACIONES

MÚLTIPLES (HSD DE TUKEY)

\begin{tabular}{|c|c|c|c|c|c|c|c|}
\hline Hipótesis & $\begin{array}{l}\text { Variable / } \\
\text { Página Web }\end{array}$ & $\begin{array}{c}\text { (I) } \\
\text { Grupo }\end{array}$ & $\begin{array}{c}(\mathbf{J}) \\
\text { Grupo }\end{array}$ & $\begin{array}{c}\text { Diferencia } \\
\text { de medias } \\
(\mathrm{I}-\mathrm{J})\end{array}$ & $\begin{array}{l}\text { Error } \\
\text { típico }\end{array}$ & Sig. & Resultado \\
\hline \multirow[t]{12}{*}{$\mathbf{H}_{3}$} & \multirow{6}{*}{$\begin{array}{l}\text { Tiempo de } \\
\text { fijación / } \\
\text { pw1 }\end{array}$} & \multirow[t]{2}{*}{$\mathrm{GE}_{1}$} & $\mathrm{GE}_{2}$ & 0.168 & 0.170 & $0.590^{\mathrm{NS}}$ & \multirow[t]{2}{*}{ ACEPTADA } \\
\hline & & & GC & 0.845 & 0.170 & $0.000 * *$ & \\
\hline & & \multirow[t]{2}{*}{$\mathrm{GE}_{2}$} & $\mathrm{GE}_{1}$ & -0.168 & 0.170 & $0.590^{\mathrm{NS}}$ & \\
\hline & & & $\mathrm{GC}$ & 0.677 & 0.176 & $0.000 * *$ & \\
\hline & & \multirow[t]{2}{*}{$\mathrm{GC}$} & $\mathrm{G}_{1}$ & -0.845 & 0.170 & $0.000 * *$ & \\
\hline & & & $\mathrm{G}_{2}$ & -0.677 & 0.176 & $0.000 * *$ & \\
\hline & \multirow{6}{*}{$\begin{array}{l}\text { Tiempo de } \\
\text { fijación / } \\
\text { Pw2 }\end{array}$} & \multirow[t]{2}{*}{$\mathrm{GE}_{1}$} & $\mathrm{GE}_{2}$ & -0.074 & 0.150 & $0.872^{\mathrm{NS}}$ & \\
\hline & & & GC & 0.822 & 0.150 & $0.000 * *$ & \\
\hline & & \multirow[t]{2}{*}{$\mathrm{GE}_{2}$} & $\mathrm{GE}_{1}$ & 0.074 & 0.150 & $0.872^{\mathrm{NS}}$ & \\
\hline & & & GC & 0.897 & 0.155 & $0.000 * *$ & \\
\hline & & \multirow[t]{2}{*}{ GC } & $\mathrm{G}_{1}$ & -0.822 & 0.150 & $0.000 * *$ & \\
\hline & & & $\mathrm{G}_{2}$ & -0.897 & 0.155 & $0.000 * *$ & \\
\hline
\end{tabular}

$* * p<0.01 ;$ NS $=$ No significativo $(p>0.05)$.

Tal como se aprecia, los tiempos de fijación medios en el área de interés de los grupos experimentales 1 y 2 , no difieren significativamente entre ellos desde un punto de vista estadístico para ninguna de las dos páginas Web $\left(p_{\mathrm{GE} 1-\mathrm{GE} 2 \mathrm{pw} 1}=0.59\right.$ y $\left.p_{\mathrm{GE} 1-\mathrm{GE} 2 \mathrm{pw} 2}=0.872\right)$, pero ambos lo hacen con respecto al grupo de control, tanto para la página Web 1 $\left(p_{\mathrm{GC}-\mathrm{GE} 1 \mathrm{pw} \mathrm{l}}=0.00\right.$ y $\left.p_{\mathrm{GC}-\mathrm{GE} 2 p w 1}=0.00\right)$ como para la página Web $2\left(p_{\mathrm{GC}-\mathrm{GE} 1 \mathrm{pw} 2}=0.00\right.$ y $p_{\mathrm{GC}-}$ $\mathrm{GE}_{2 \mathrm{pw}}=0.00$ ) por lo que no es posible rechazar la $\mathrm{H}_{3}$ y se afirma que: la incorporación de elementos gráficos no relacionados con la empresa en sí misma incrementa el tiempo de fijación de la mirada sobre los mismos y distrae la atención del consumidor.

En la Tabla 8 se exhiben, en forma de mapas de calor, las representaciones gráficas correspondientes a todas las observaciones de los grupos de estudio para cada página Web, señalando (con un cuadro) el área en la que fueron realizadas las manipulaciones, es decir, el área de interés a la que se refieren los párrafos y tablas anteriores. A través de ella es posible apreciar las diferencias previstas del comportamiento de la atención de la mirada de los usuarios; nótense especialmente las diferentes concentraciones entre los resultados del GC con respecto a los otros dos grupos. 
Tabla 8

MAPAS DE CALOR GLOBALES POR GRUPO Y PÁGINA WEB

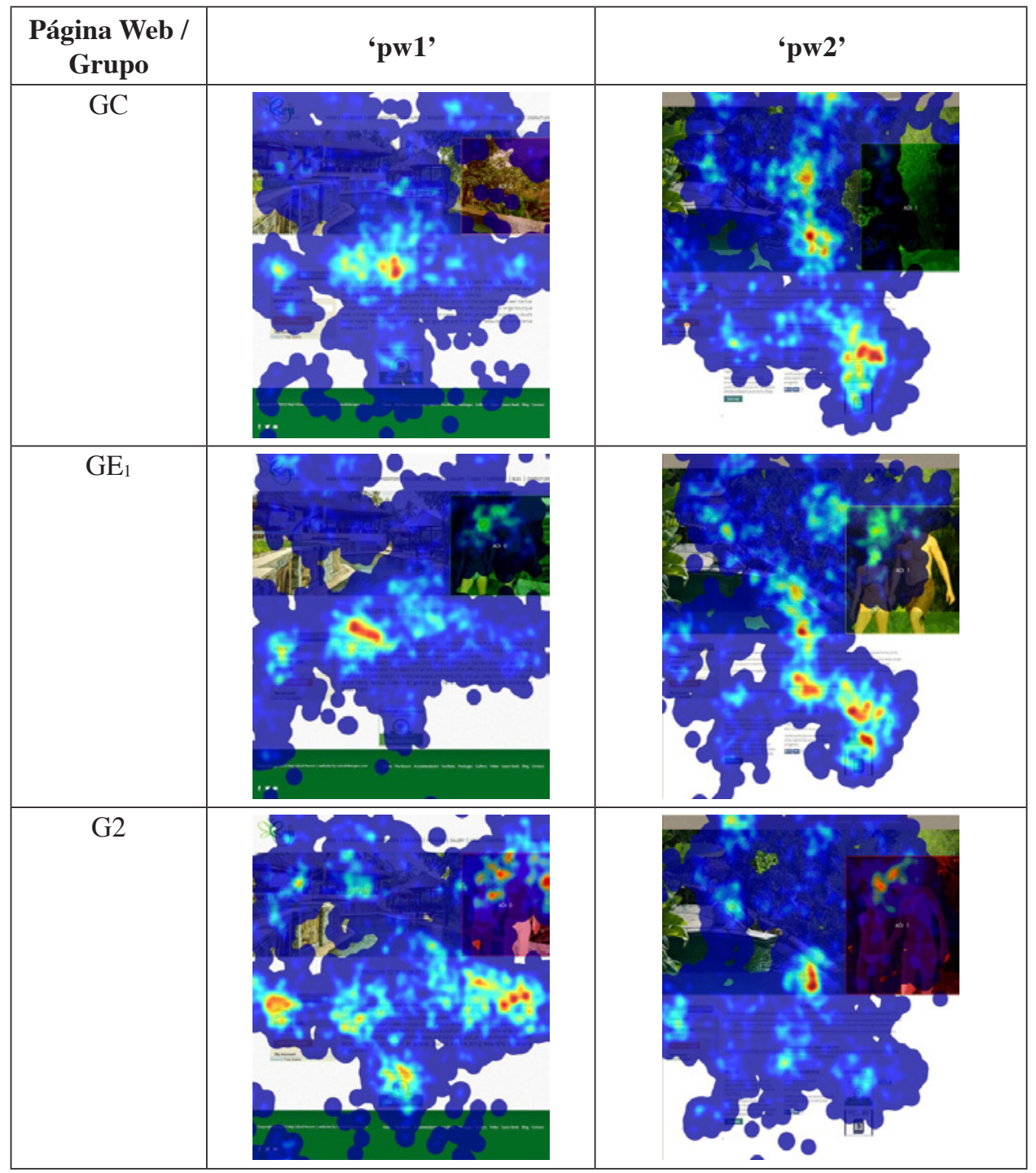

\subsection{Discusión de los resultados}

En el presente epígrafe se discuten los resultados obtenidos en esta investigación a la luz de la revisión de la literatura. En primer lugar, la aceptación de $\mathrm{H}_{1}$, y la consecuente confirmación de que el atractivo visual declarado por usuarios de la Generación Y de una página Web de promoción de establecimientos de alojamiento turístico no difiere signifi- 
cativamente por la presencia de celebridades o de personas no célebres en su diseño, se alinea con el enfoque basado en la teoría Gestalt (Arnheim, 1988, 1992) e identificado por Djamasbi et al. (2010) como útil para el estudio de las respuestas estéticas a partir de estímulos completos, en el sentido de que las percepciones de los individuos acerca de un objeto determinado, en este caso páginas Web, son globales.

Tal como fue previsto, la evidencia empírica analizada refuta estadísticamente los planteamientos teóricos de Djamasbi et al. (2010) en cuanto a la supuesta existencia de una relación de dependencia entre la presencia de celebridades en el estímulo y su atractivo visual, contradiciendo su afirmación acerca de que la presencia de celebridades en páginas Web sea una característica que influya favorablemente en la respuesta estética de los miembros de la Generación Y hacia la misma, al menos en el contexto de este trabajo.

El hecho de que la media del atractivo visual resultara estadísticamente igual para los tres grupos y para las dos páginas Web empleados en este experimento sugiere, en concordancia con los postulados de Williams y Page (2011) que, en efecto, los miembros de la Generación Y son fuertemente influenciados por el uso de imágenes y que sus primeras impresiones de una página Web pueden estar basadas en el uso de elementos visuales (Robins y Holmes, 2008).

En segundo lugar, la aceptación de $\mathrm{H}_{2}$ y por consiguiente de la afirmación acerca de que la credibilidad declarada por usuarios de la Generación Y de una página Web de promoción de establecimientos de alojamiento turístico no difiere significativamente por la presencia de celebridades o de personas no célebres en su diseño, soporta los planteamientos de Sundar (2008) en el sentido de que la credibilidad de los miembros de la Generación Y se sustenta en sus juicios sobre la fuente y el contenido del mensaje, es decir, en el ethos y el logos (Metzger y Flanagin, 2013), y refutan el postulado de Fogg et al. (2003) acerca de que la credibilidad de las páginas Web se base más en los elementos visuales contenidos en sus diseños que en el propio contenido o fuente de la información. En este mismo tenor, los resultados de la presente investigación no compadecen los argumentos de Cyr et al. (2009) en relación a que la presencia de personas en páginas Web pueda influir en la credibilidad en entornos virtuales. Este hallazgo cobra relevancia ya que, tal como señalan Williams y Page (2011), los miembros de la Generación Y valoran en gran medida la verdad, la integridad y la honestidad.

En tercer lugar, en lo referente a la contrastación y aceptación de $\mathrm{H}_{3}$, los resultados obtenidos mediante el aprovechamiento del segundo enfoque para el estudio de las respuestas estéticas identificado en la literatura por Djamasbi et al. (2010) -el experimentalsoporta la idea de que es posible descubrir, de una mejor manera, las percepciones de los sujetos a través de los efectos producidos por partes aisladas de un objeto, en este caso, las páginas Web (Martindale et al., 1990; Swede, 1994), gracias a la complementariedad de los enfoques Gestalt y estética experimental (Djamasbi et al., 2010).

Así mismo, al corroborar que la incorporación de elementos gráficos no relacionados con la empresa en sí misma incrementa el tiempo de fijación de la mirada sobre ellos distrayendo la atención del consumidor, refuerza el planteamiento acerca de que la utilización de tecnologías de rastreo ocular posibilita un mejor aprovechamiento de los elementos funcionales y hedónicos que componen una página Web para crear ventajas competitivas e incrementar el valor de la empresa (Djamasbi et al., 2007). Sin embargo, en línea con 
las ideas de Wang (2007), Zhang (2000) y Huang (2003) -y tal como se demostró-, la presencia de personas, célebres o no, en una página Web de promoción de establecimientos de hospedaje puede constituir un elemento distractor capaz de conducir a los usuarios de la Generación Y a destinarle parte significativa de su atención, afectando negativamente la efectividad del mensaje publicitario.

En términos más amplios, los hallazgos de esta investigación permiten deducir que la presencia de celebridades no solamente no contribuye positivamente a elevar el atractivo visual o la credibilidad de sitios Web de promoción de establecimientos de alojamiento turístico enfocadas en la Generación Y, sino que inclusive distrae la atención de otros elementos contenidos en su diseño que interesan más a la empresa para promover sus productos y servicios.

En efecto, los resultados sugieren que el valor atribuido a las celebridades como fuente de atractivo visual y credibilidad para los sujetos de la Generación Y es, al menos en el contexto de este estudio, infundado y aún contraproducente, por lo que se considera más conveniente para las empresas interesadas en captar la atención y confianza de los usuarios de esa generación que no incluyan celebridades en sus diseños Web por dos razones principales. La primera es que las inversiones de producción y regalías requeridas para utilizar la imagen de las celebridades suelen ser cuantiosas y, como en este caso, ineficientes por lo que no se justifican; y la segunda, que su presencia en la página Web reduce el tiempo efectivo que el usuario dedica a observar información propia de la empresa y sus ofrecimientos, reduciendo sus oportunidades de brindar información relevante de la organización.

\section{CONCLUSIONES, LIMITACIONES Y LÍNEAS DE INVESTIGACIÓN FUTURA}

En un entorno global crecientemente competitivo, con medios de comunicación más variados, complejos, fragmentados y saturados que nunca antes, y con consumidores cada vez más selectivos, exigentes, sofisticados y renuentes a la publicidad, el aprovechamiento de las TIC con fines mercadológicos en general y de promoción turística en particular resulta indispensable para aquellas organizaciones que deseen mantener y/o incrementar su mercado atendido. Los sitios Web siguen siendo una de las principales herramientas para acceder directamente a los consumidores, especialmente al importante segmento para la comercialización de productos y servicios que representan los miembros de la Generación Y.

En la literatura especializada se ha planteado que la presencia de celebridades constituye una de las características esenciales para incrementar tanto el atractivo visual como la credibilidad de las páginas Web. Pretendiendo contribuir al conocimiento del marketing turístico, y con el objetivo de contrastar empíricamente esos postulados, se llevó a cabo una investigación causal basada en un experimento verdadero para un factor 3 x 2 solo post test en el cual se manipuló la presencia/ausencia de celebridades y de personas no célebres como factor independiente y se midieron y analizaron sus efectos en las variables dependientes: atractivo visual, credibilidad y tiempo de fijación de la mirada. Los resultados refutaron dichas ideas y se comprobó que la presencia de celebridades no aumenta 
el atractivo ni credibilidad de las páginas Web, sino que, sorprendentemente y por el contrario, resta la atención dedicada a la información de las propias empresas por lo que su valor como fuente de atractivo visual y credibilidad resulta, en el mejor de los casos, nulo.

El conocimiento generado en esta investigación contribuye al estudio del marketing turístico de tres formas. En primer lugar, hasta donde llega nuestro conocimiento, es la primera vez que se contrastan empíricamente, mediante un enfoque doble, desde una perspectiva causal y a través de un experimento verdadero, las reacciones estéticas de los miembros de la Generación Y relacionadas con la presencia de celebridades en las páginas Web como fuente de atractivo visual y credibilidad. En segundo lugar, este estudio es pionero en el uso de herramientas de rastreo ocular para la investigación científica del marketing en Latinoamérica y del marketing turístico en particular. En tercer lugar, la refutación de planteamientos teóricos previos ayuda a sentar bases para futuros estudios de académicos y profesionales al respecto, tales como las que se señalan más adelante.

Desde el punto de vista gerencial, los hallazgos de esta investigación constituyen evidencia estadística que puede ser tomada como referencia por parte de los tomadores de decisiones, al momento de diseñar las páginas Web de promoción, particularmente de promoción de alojamientos turísticos enfocados a los miembros de la Generación Y.

Como cualquier otra investigación, el presente trabajo no está exento de limitaciones que restringen la generalización de sus resultados, por lo que ésta debe ser hecha con prudencia. La primera limitación se debe a que todos los participantes fueron estudiantes universitarios de Cozumel, Quintana Roo, México, quienes si bien fueron seleccionados y asignados a los grupos de estudio al azar y por sus edades pertenecen a la Generación Y, no necesariamente son representativos de todos los miembros de dicho segmento de mercado, por tal motivo se requiere y recomienda replicar esta investigación en contextos distintos.

La segunda limitación se deriva de que únicamente se emplearon páginas Web de promoción de servicios de alojamientos turísticos exóticos, los cuales son productos de alta implicación, y la tercera tiene que ver con el número de páginas Web empleadas en el estudio -sólo dos. Luego, también es necesario realizar nuevas investigaciones en sectores económicos distintos al turismo y/o con bienes y/o servicios de alta y baja implicación, así como empleando distintas páginas de promoción.

\section{REFERENCIAS}

ARNHEIM, R. (1988): «Visual dynamics». American Scientist, vol. 76 (6), pp. 585-591. ARNHEIM, R. (1992): «But is it science», en Emerging Visions of the Aesthetic Process: Psychology, Semiology, and Philosophy. Cambridge University Press, Cambridge, pp 27-36.

BAKEWELL, C. y MITCHELL, V.W. (2003): «Generation Y female consumer decisionmaking styles». International Journal of Retail and Distribution Management, vol. 31 (2), pp. 95-106.

BARBER, T., BERTRAND, J., CHRIST, C., MELLOY, B. y NEYENS, D. (2014): «Comparing the Use of Active versus Passive Navigational Tools In a Virtual Desktop Environment via Eye Tracking». En Proceedings of the Human Factors and Ergonomics Society Annual Meeting, pp. 1954-1958. 
BIGNÉ ALCAÑIZ, E. (1996): «Turismo y marketing en España. Análisis del estado de la cuestión y perspectivas de futuro». Estudios Turísticos, n 129, pp. 105-127.

BUHALIS, D. y LAW, R. (2008): «Progress in information technology and tourism management: 20 years on and 10 years after the Internet-The state of eTourism research». Tourism Management, vol. 29 (4), pp. 609-623.

BUSCHER, G., CUTRELL, E., y MORRIS, M.R. (2009). «What do you see when you $>$ re surfing?: using eye tracking to predict salient regions of Web pages». En Proceedings of the SIGCHI conference on human factors in computing systems, pp. 21-30. ACM.

BUSCHER, G., DUMAIS, S.T. y CUTRELL, E. (2010): «The good, the bad, and the random: an eye-tracking study of ad quality in Web search». En Proceedings of the 33rd international ACM SIGIR conference on research and development in information retrieval, pp. 42-49. ACM.

CUTRELL, E., y GUAN, Z. (2007): «What are you looking for?: an eye-tracking study of information usage in Web search». En Proceedings of the SIGCHI conference on human factors in computing systems, pp. 407-416. ACM.

CUI, Y., TRENT, E.S., SULLIVAN, P.M. y MATIRU, G.N. (2003). «Cause-related marketing: How generation Y responds». International Journal of Retail \& Distribution Management, vol. 31 (6), pp. 310-320.

CYR, D. y HEAD, M. (2013): «The impact of task framing and viewing timing on user Website perceptions and viewing behavior». International Journal of Human-Computer Studies, vol. 71(12), pp. 1.089-1.102.

CYR, D., HEAD, M., LARIOS, H. y PAN, B. (2009): «Exploring human images in Website design: a multi-method approach». MIS Quarterly, vol. 33 (3), pp. 539-566.

DEL PINO ROMERO, C. y OLIVARES, F. (2007): «Brand placement y advertainment: Integración y fusión entre la ficción audiovisual y las marcas». Zer-Revista de estudios de comunicación, $\mathrm{n}^{\mathrm{O}}$ 22, pp. 341-367.

DENG, J., KING, B. y BAUER, T. (2002): «Evaluating natural attractionsfortourism». Annals of Tourism Research, vol. 29 (2), pp. 422-438.

DJAMASBI, S., SIEGEL, M. y TULLIS, T. (2010): «Generation Y, Web design, and eye tracking». International Journal of Human-Computer Studies, vol. 68 (5), pp. 307-323.

DJAMASBI, S. et al. (2007): «Gender preferences in Web design: usability testing through eye tracking». AMCIS 2007 Proceedings, pp. 1-8.

DJAMASBI, S. et al. (2008): «Generation Y and Web design: Usability through eye tracking». AMCIS 2008 Proceedings, pp. 1-11.

DUMAIS, S.T., BUSCHER, G. y CUTRELL, E. (2010): «Individual differences in gaze patterns for Web search». En Proceedings of the third symposium on information interaction in context, pp. 185-194. ACM.

ECHTNER, C.M. y JAMAL, T.B. (1997): «The Disciplinary Dilemma of Tourism Studies». Annals of Tourism Research, vol. 24 (4), pp. 868-883.

FONG, S., TSANG, W., CHENG, Y., KI, W., MA, A. y MACFARLANE, D. (2015): «Single-channel Electroencephalographic Recording in Children with Developmental Coordination Disorder: Validity and Influence of Eye Blink Artifacts». Journal of Novel Physiotherapies, vol. 5 (4), pp. 1-6. 
FOGG, B.J., SOOHOO, C., DANIELSON, D.R., MARABLE, L., STANFORD, J. y TAUBER, E.R. (2003): «How do users evaluate the credibility of Web sites? A study with over 2,500 participants». En Proceedings of the 2003 conference on Designing for user experiences. ACM, pp. 1-15.

FORO ECONÓMICO MUNDIAL (2014): «Reporte Global de las Tecnologías de Información». Bilbao-Osorio, B., Dutta, S. y Lanvin, B (Ed.).

GUAN, Z., y CUTRELL, E. (2007): «An eye tracking study of the effect of target rank on Web search». En: Proceedings of the SIGCHI conference on human factors in computing systems, pp. 417-420. ACM.

GUTTENTAG, D.A. (2009): «Virtual Reality: Applications and Implications for Tourism». Tourism Management, vol. 31 (5), pp. 637-651.

HERRERO CRESPO, A. y SAN MARTÍN GUTIÉRREZ, H. (2012): «Influence of the user's psychological factors on the online purchase intention in rural tourism: Integrating innovativeness to the UTAUT framework». Tourism Management, vol. 33 (2), pp. 341-350.

HUANG, M.H. (2003): «Designing Website Attributes to Induce Experiential Encounters». Computers in Human Behavior, vol. 19 (4), pp. 425-442.

JACOB, R.J.K. y KARN, K.S. (2003): «Eye tracking in human-computer interaction and usability research: Ready to deliver the promises». En The mind's eye: Cognitive and applied aspects of eye movement research. Amsterdam, Elsevier Science, pp. 573-605.

JACOBY, J. (2002): «Stimulus-Organism-Response Reconsidered: An Evolutionary Step in Modeling (consumer) Behavior». Journal of Consumer Psychology, vol. 12 (1), pp. 51-57.

KOTLER, P. (2011): «Reinventing marketing to manage the environmental imperative». Journal of Marketing, vol. 75 (4), pp. 132-135.

LAI, M. L. et al. (2013): «A review of using eye-tracking technology in exploring learning from 2000 to 2012». Educational Research Review, n 10, pp. 90-115.

LAVIE, T. y TRACTINSKY, N. (2004): «Assessing dimensions of perceived visual aesthetics of Web sites». International Journal of Human-Computer Studies, vol. 60 (3), pp. 269-298.

LEASK, A., BARRON, P., ENSOR, J. y FYALL, A. (2014): Generation Y: The Impact of Generational Changes in Consumer Behaviour on the Marketing of Tourist Attractions. Working Paper. Edinburgh Napier University, Edinburgh.

LINDGAARD, G. (2007): «Aesthetics, visual appeal, usability and user satisfaction: What do the user's eyes tell the user's brain?». Australian Journal of Emerging Technologies and Society, vol.5 (1), pp. 1-14.

LINDGAARD, G., FERNANDES, G., DUDEK, C. y BROWN, J. (2006): «Attention Web designers: You have 50 milliseconds to make a good first impression!». Behavior and informationtechnology, vol. 25 (2), pp. 115-126.

MARTÍ PARREÑO, J., ALDÁS MANZANO, J., CURRÁS PÉREZ, R. y SÁNCHEZ GARCÍA, I. (2010): «El emplazamiento de producto: conceptualización, nuevos formatos y efectos sobre el consumidor». Teoría y Praxis, vol. 1 (8), pp. 113-136. 
MARTINDALE, C., MOORE, K. y BORKUM, J. (1990): «Aesthetic preference: anomalous findings for Berlyne's psychobiological theory». American Journal of Psychology, vol. 103 (1), pp. 53-80.

MARTÍNEZ, J. (2013): «La gestión de la empresa turística centrada en el residente. El caso de la Generación Y». Turydes, vol. 6 (15), pp. 1-17.

MATHWICK, C., MALHOTRA, N. y RIGDON, E. (2001): «Experiential Value: Conceptualization, Measurement and Application in the Catalog and Internet Shopping Environment». Journal of Retailing, vol. 77 (1), pp. 39-56.

MATHWICK, C., MALHOTRA, N. y RIGDON, E. (2002): «The Effect of Dynamic Retail Experiences on Experiential Perceptions of Value: An Internet and Catalog Comparison». Journal of Retailing, vol. 78 (1), pp. 51-60.

METZGER, M. J. y FLANAGIN, A. J. (2013): «Credibility and trust of information in online environments: The use of cognitive heuristics». Journal of Pragmatics, no. 59, pp. 210-220.

PITTA, D.A. (2011): «Location-based social networking and marketing». Journal of Consumer Marketing, vol. 28 (2).

RAYNER, K. (1998): «Eye movements in reading and information processing: 20 years of research». Psychological Bulletin, vol. 124(3), pp. 372-422.

REPORTE ALEXA (2013): «The top 500 sites on the Web». Disponible en: http://www. alexa.com/topsites

RITCHIE, J.R. y CROUCH, G.I. (2000): «The competitive destination: A sustainability perspective». Tourism Management, vol. 21 (2), pp. 1-7.

ROBINS, D. y HOLMES, J. (2008): «Aesthetics and credibility in Web site design». Information Processing and Management, vol. 44(1), pp. 386-399.

ROTH, S., TUCH, A., MEKLER, E., BARGAS-AVILA, J. y OPWIS, K. (2013): «Location matters, especially for non-salient features-An eye-tracking study on the effects of Web object placement on different types of Websites». International Journal of Human-Computer Studies, vol. 71(3), pp. 228-235.

ROY, S., GUHA, A. y BISWAS, A. (2015): «Celebrity endorsements and women consumers in India: how generation-cohort affiliation and celebrity-product congruency moderate the benefits of chronological age congruency». Marketing Letters, vol. 26 (3), pp. 363-376.

RUBIO GIL, M.A. (2003): «El turismo del futuro: agente de conservación y desarrollo». Saberes, vol. 1, pp. 1-13.

SANCHO, A. (2006): Introducción al Turismo. Madrid, Organización Mundial del Turismo (OMT).

SHEAHAN, P. (2005): Generation Y: Thriving and surviving with generation $Y$ at work. Australia, Hardy Grant.

SINCLAIR, J. e IRANI, T. (2005): «Advocacy advertising for biotechnology: The effect of public accountability on corporate trust and attitude toward the ad». Journal of Advertising, vol. 34 (3), pp. 59-73.

SUNDAR, S.S. (2008): «The MAIN model: A heuristic approach to understanding technology effects on credibility». Digital media, youth, and credibility, en The John D. and Catherine T. MacArthur Foundation Series on Digital Media and Learning, Cambridge, pp. 73-100. 
SWEDE, G. (1994): «Basic theoretical issues». En The Psychology of Art: An Experimental Approach. Canadian Scholars' Press Inc, Toronto.

TANG, L.R., JANG, S.S. y MORRISON, A. (2012): «Dual-route communication of destination Websites». Tourism Management, vol. 33 (1), pp. 38-49.

TORRANO, J. y FLORES, E. (2007): «Factores determinantes de la actitud hacia el productplacement». En El comportamiento de la empresa ante entornos dinámicos: XIX Congreso Anual y XV Congreso Hispano Francés de AEDEM, vol. 2, pp. 1-19.

TRACTINSKY, N. (2004): «Toward the study of aesthetics in information technology». ICIS 2004 Proceedings, vol. 62, pp. 771-780.

WANG, K. C. et al. (2007): «More Information, Stronger Effectiveness? Different Group Package Tour Advertising Components on Web Page». Journal of Business Research, vol. 60 (4), pp. 382-387.

WATERS, J. (2006): «Young, with tons of purchasing power». Market Watch, The Wall Street Journal Digital Network, Chicago.

WILLIAMS, K.C. y PAGE, R.A. (2011): «Marketing to the generations». Journal of Behavioral Studies in Business, vol. 3 (1), pp. 37-53.

ZHANG, P. (2000): «The effects of animation on information seeking performance on the World Wide Web: Securing attention or interfering with primary tasks?». Journal of the Association for Information Systems, vol. 1 (1), pp. 1-28.

ZUGAL, S. y PINGGERA, J. (2014): «Low-Cost Eye-Trackers: Useful for Information Systems Research?». En: Advanced Information Systems Engineering Workshops (LAZAROS, I.; MICHAEL, P. y KLAUS, P.) (Ed.), vol. 178, pp. 159-170. Springer International Publishing. 
